\title{
A view into the core of $\alpha$ Cen A
}

\author{
Michaël Bazot ${ }^{1, \star}$, Jørgen Christensen-Dalsgaard², Othman Benomar ${ }^{1}$, and Laurent Gizon ${ }^{3,4}$ \\ ${ }^{1}$ Center for Space Science, NYUAD Institute, New York University Abu Dhabi, PO Box 129188, Abu Dhabi, United Arab Emirates \\ ${ }^{2}$ Stellar Astrophysics Centre, Department of Physics and Astronomy, Aarhus University, Ny Munkegade 120, DK-8000 Aarhus C, \\ Denmark \\ ${ }^{3}$ Max-Planck-Institut für Sonnensystemforschung, 37077 Göttingen, Germany \\ ${ }^{4}$ Institut für Astrophysik, Georg-August-Universität Göttingen, 37077 Göttingen, Germany
}

\begin{abstract}
We present results of modelling of $\alpha$ Cen A. In order to estimate the physical parameters of this star, we modelled spectroscopic, interferometric, astrometric and asteroseismic data. To that effect we chose to use a Bayesian approach to parameter estimation, which allowed us, in particular, to define our prior knowledge on the parameters. An important question we wanted to address was to assess whether or not $\alpha$ Cen A has a convective core. We found that the data we used give indecisive results on this issue. If the star has a convective core, and provided that overshooting is taken into account, there is a possibility for the star to be in the peculiar state in which the ppII chain is the main driver of nuclear energy generation. We also found a non-negligible probability for $\alpha$ Cen A to be a very early subgiant.
\end{abstract}

\section{Introduction}

$\alpha$ Cen A has long been an attractive object for stellar physicists. It is the closest star to Earth and very precise data have been collected from interferometry or spectroscopy, in particular ground-based asteroseismology. Moreover, it is part of a relatively close binary, which gives us invaluable information on its mass. However, as of today, there were still uncertainties as to the state of its central regions. A outstanding issue is whether or not this star possesses a convective core. In this study, we aim at assessing rigorously our current state of knowledge about the central regions of $\alpha$ Cen $\mathrm{A}$, given the current observational data, using numerical Bayesian statistical methods to conduct a (probabilistic) modelling of the star.

\section{Method}

\subsection{Bayesian estimation}

The goal of Bayesian Statistics is to obtain estimates of the parameters of some model, which we will call in the following a Bayesian statistical model. In mathematical terms, this model is described by what we will call a Posterior Density Function (PDF), $\pi(\theta \mid X)$, with $\theta$ the parameters of the model and $X$ some observational data. It is key to understand that in a Bayesian statistical model the parameters are the random quantities, whereas the data are in a frequentist approach. The data are distributed according to a density $\pi(X \mid \theta)$, which is called a likelihood if we consider the data as a fixed quantity and let the parameters

\footnotetext{
^e-mail: mb6215@nyu.edu
}

vary. Being given such a likelihood, one needs to define a prior density on the parameters, $\pi(\theta)$, in order to define completely a Bayesian statistical model. These densities are related through Bayes' formula

$$
\pi(\theta \mid X) \propto \pi(\theta) \pi(X \mid \theta) .
$$

In general, whether one is using the frequentist or the Bayesian approach, observations and a parametric model are needed to define a statistical model. This is enough to construct a likelihood, but a Bayesian statistical model has to be supplemented by the definition of prior densities.

\subsubsection{Data}

We make use of various data collected throughout the literature. $\alpha$ Cen A being the closest star to our Sun, there exists a huge amount of high-quality observations, be it in astrometry, spectroscopy, interferometry, photometry or asteroseismology. In the present study we used the effective temperature and luminosity as referenced by Eggenberger, Charbonnel, Talon et al. [1], the radius published by Kervella et al. [2] and the metallicity adopted by Thoul et al. [3]. These are the quantities forming the vector $X$ in the space of observations. We used the seismic data obtained from a 5-night HARPS run [4]. From the 34 frequencies, we were able to obtained 6 small separations $\delta_{n, l=0}=v_{n, l=0}-v_{n-1, l=2}$.

Measurements of the stellar mass also exist for $\alpha$ Cen A since it forms a close binary together with $\alpha$ Cen B. Having a period of $\sim 80$ years, the system has been observed for over a complete revolution and as such is known with good precision. The value of the mass used here is from 
Pourbaix et al. [5]. This will enter our model through the prior density.

Table 1: Priors used for the Bayesian statistical model presented in this paper. The first column gives the parameter (mass, age, initial metallicity, initial hydrogen mass fraction, mixing-length parameter, overshoot parameter), the second column gives the functional form of the prior. The last column gives the relevant parameters of the prior (upper and lower bounds, the mean and standard deviation are given for the truncated Gaussian).

\begin{tabular}{lcr}
\hline Parameter & Prior & Dist. parameters \\
\hline$M\left(\mathrm{M}_{\odot}\right)$ & Truncated Gaussian & {$[1.07,1.13,1.105$,} \\
& & $\left.7 \times 10^{-3}\right]$ \\
$t_{\star}(\mathrm{Gyr})$ & Uniform & {$[1,8]$} \\
$Z_{0}$ & Uniform & {$[0.01,0.04]$} \\
$X_{0}$ & Uniform & {$[0.6,0.75]$} \\
$\alpha$ & Uniform & {$[1.2,2.6]$} \\
$\alpha_{\mathrm{ov}}$ & Discrete/Uniform & $0 /[0,0.75]$ \\
\hline
\end{tabular}

\subsubsection{Physical model}

To model these observations, we used the Aarhus Stellar Evolution Code [6]. It assumes spherical symmetry for the star and neglects the effect of magnetic fields, rotation, wave-induced mixing, mass-loss or radiative levitation.

We chose to use the OPAL 95 opacity tables supplemented by low-temperature values given by Ferguson et al. [7]. The OPAL 2005 equation of state [8] was adopted. The relative abundances from Grevesse \& Noels [9] and the NACRE reaction rates [10] were selected. Convection is described using the classical framework of the mixinglength theory [11], which implies the tuning of a proportionality coefficient $\alpha$ between the mean-free path of convective elements and, as implemented in ASTEC, the characteristic pressure scale height.

In addition to this mixing-length parameter, other parameters have to be given as input to ASTEC. In our case, we will also consider the mass, the age, the initial hydrogen mass fraction and metallicity, and, in some cases, a core-overshoot parameter $\alpha_{\text {ov }}$ controlling the amount of additional mixing occurring above potential convective cores. These form the vector $\theta$ in the parameter space.

\subsubsection{Bayesian statistical model}

The last step in defining our Bayesian statistical model consists in defining our likelihood and priors on the parameters. The former is taken to be a multivariate Gaussian. Furthermore, we made the assumption of independent observations, hence reducing the non-zero entries of the covariance matrix to its diagonal components.

The type of prior chosen is given parameter by parameter in Table 1. For each of them we give the lower and upper boundaries of the definition domain. For the truncated Gaussian, these are followed by its mean and standard deviation.

Our model was then sampled using an adaptive Markov chain Monte Carlo (MCMC) algorithm [12].

\subsection{Results}

After sampling the PDF for $\theta$, we were able to estimate the marginal densities of the individual parameters (by integrating over all the others) but also the PDFs for other functions of the parameters.
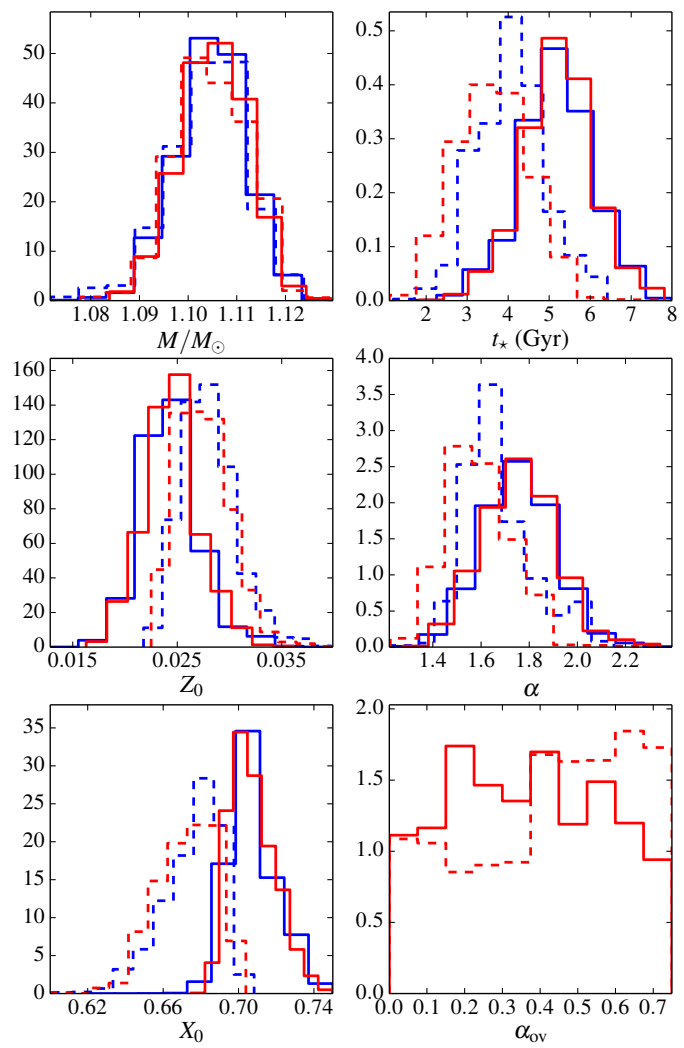

Figure 1: Marginal posterior densities for models without (full lines) and with (dashed lines) convective cores. In blue are shown the results for models without overshoot and in red with overshoot.

The estimates of the stellar parameters are given in Table 2. Interestingly enough the inclusion of overshooting does not impact much the estimates of the parameters. Since many models in our MCMC simulations show convective cores, which hence should be affected by overshoot processes, this hints to some liberty left by the seismic data on the constraining of the stellar innermost regions. To strengthen this picture, one can note that including overshoot does not dramatically increase the odds of $\alpha$ Cen A having a convective core. It simply increases its average radius. In terms of PDFs, this translate into the observation of two potential regimes of solutions that could both reproduce the observational constraints.

These are shown in Fig. 1. There we can see that the convective-core models are, on average, younger. They also have a lower initial hydrogen mass fractions and mixing-length parameters, and higher initial metallicities. 

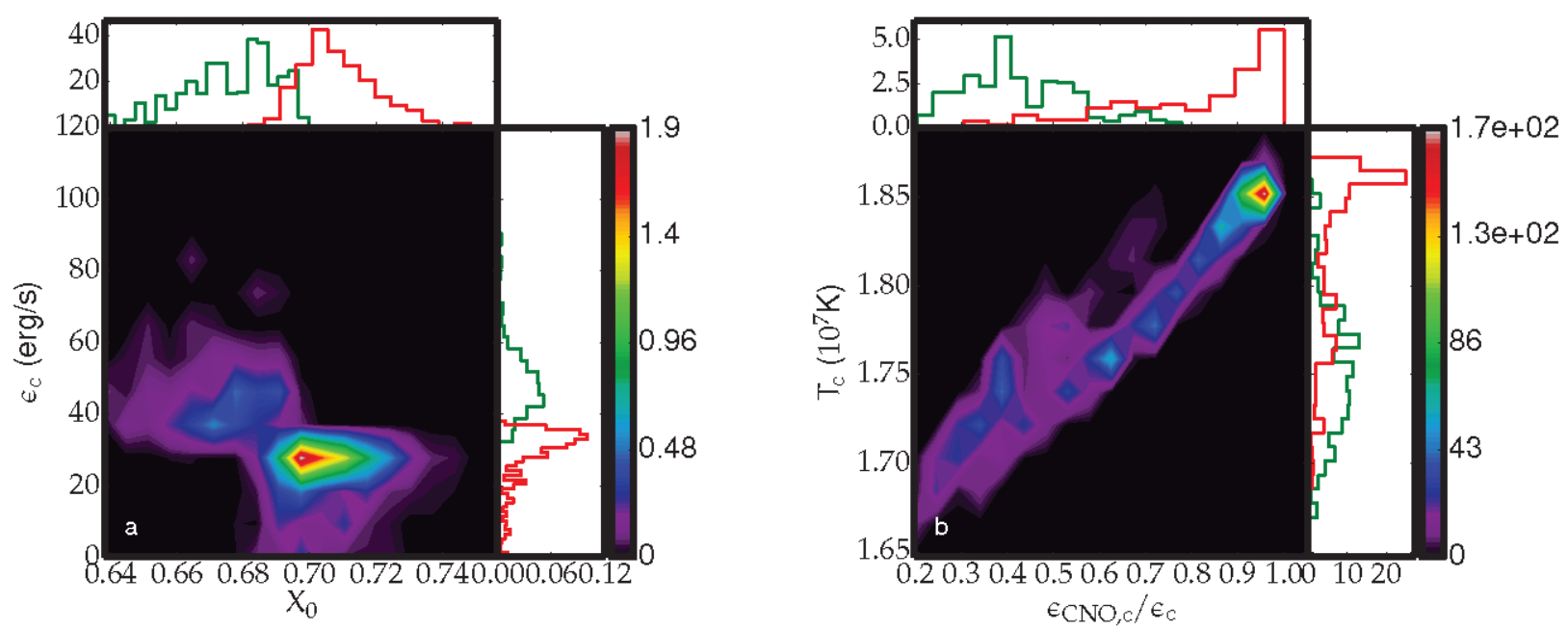

Figure 2: Posterior joint PDFs for (a) $X_{0}$ and $\varepsilon_{0}$ the central energy generation rate, (b) $\varepsilon_{\mathrm{CNO}, \mathrm{c}} / \varepsilon_{0}$, the relative rate of CNO energy generation rate at the centre, and $T_{\mathrm{c}}$, the central temperature. These distributions have been obtained from the second run in Table 2 . The smaller panels display the marginal PDFs for each individual quantity. Two populations are distinguished, in red the radiative-core models and in green the convective-core models.

These two solutions obtained for $\alpha, X_{0}, Z_{0}$ correspond to different positions on the zero-age main sequence. Basically, ZAMS progenitors for convective-core models of $\alpha$ Cen $\mathrm{A}$ are, again on average, more luminous and hotter than their radiative-core counterparts. The age is then simply an adjustment parameter mostly constrained by the need to reproduce the luminosity of the star.

A look at Fig. 2 gives a more detailed picture of the centre of $\alpha$ Cen A. In the left panel is plotted the joint probability density of the initial hydrogen mass fraction and the energy generation rate at the centre of the star. These are for models that include overshooting. As stated above, the models with and without convective cores represent two very distinct populations in terms of initial hydrogen content. This is equally the case for the energy generation rate, and we can clearly see the two above mentioned solutions appear. The models with convective cores have the largest energy production rate at the centre, as expected.

However, the overall picture of a potential convective core in models with overshoot does not exactly match the classical one, i.e. the CNO cycle becoming dominant and, due to the larger sensitivity of this process on temperature, giving rise to large energy flux near the centre. The right panel of Fig. 2 shows the joint probability density for the ratio of the $\mathrm{CNO}$ and total energy generation rates and the temperature, all evaluated at the centre of the star. Again, the two solutions to the estimation problem are seen clearly. However, the striking fact is that radiativecore models are, on average, hotter at the centre and dominated by the CNO cycle whereas the convective-core models are mostly powered by the ppII burning chain.

This phenomenon is not unknown and has already been noted by [13, p. 479], in the case of a $2 \mathrm{M}_{\odot}$ without overshooting for which a convective core develops before the CNO cycle takes over. Note, however, that this does not mean that the CNO cycle is unimportant, it must

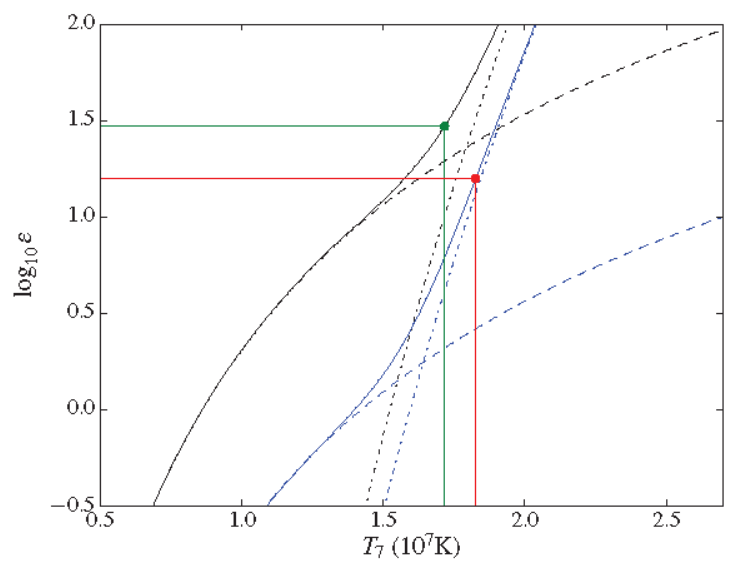

Figure 3: Energy generation rates as functions of the temperature computed for the best a posteriori models with a radiative core (blue lines) and convective core (black lines). The full lines represent the total energy generation rates, the dashed lines the pp-chain rates and the dot-dashed line the $\mathrm{CNO}$ rates. The green and red dots mark the position of the centre points of the best convective-core and radiative-core model in the plane.

contribute non-negligibly to the energy generation rate in order for a convective core to develop.

A simple explanation in our case for this picture to occur is related to the fact that $\alpha$ Cen A, regardless of it having a convective core, lies in a region of the parameter space close to the transition from the pp to the CNO hydrogen-burning process. In this case, since these do not only depend on the temperature but also on the hydrogen abundances, a difference in chemical composition at the centre can therefore account for a hydrogen-rich ppII-dominated core with higher energy generation than 
Table 2: Estimated parameters of $\alpha$ Cen A from different MCMC simulations without (top) and with (bottom) convective-core overshoot. Column 1-6 give the estimates as the posterior modes of the PDFs for the mass, age, initial metallicity and hydrogen massfraction, mixing-length and, if included, overshoot parameter. The upper and lower bounds of associated credible intervals are also given. Column 7 shows the odds for central convective onset. Column 8 gives the estimated fractional radius of the convective core (taken as the full extent of the mixing-homogenized region in case of overshoot) of $\alpha$ Cen A, if present.

\begin{tabular}{cccccccc}
\hline$M\left(\mathrm{M}_{\odot}\right)$ & $t_{\star}(\mathrm{Gyr})$ & $Z_{0}$ & $X_{0}$ & $\alpha$ & $\alpha_{\mathrm{ov}}$ & $\%$ Convective cores & $r_{\mathrm{cc}} / R_{\star}$ \\
\hline $1.106_{-0.008}^{+0.008}$ & $4.7_{-1.0}^{+1.2}$ & $0.025_{-0.003}^{+0.004}$ & $0.70_{-0.02}^{+0.02}$ & $1.68_{-0.13}^{+0.19}$ & - & 37 & $0.038_{-0.013}^{+0.011}$ \\
$1.105_{-0.007}^{+0.009}$ & $4.9_{-1.5}^{+1.0}$ & $0.025_{-0.003}^{+0.004}$ & $0.70_{-0.02}^{+0.02}$ & $1.70_{-0.20}^{+0.16}$ & $0.51_{-0.35}^{+0.15}$ & 41 & $0.084_{-0.025}^{+0.020}$ \\
\hline
\end{tabular}

a hydrogen-poor mostly powered by the CNO cycle. This is what is shown in Fig. 3 where simple formulations of the energy generation rates [see e.g. 14] have been plotted for the central hydrogen mass fraction of the most probable radiative-core and convective-core models. At their centres, these have, respectively, $\rho=209 \mathrm{~g} . \mathrm{cm}^{-3}$, $X=0.11, X_{\mathrm{CNO}}=0.0062$, and $\rho=119 \mathrm{~g} . \mathrm{cm}^{-3}, X=0.44$, $X_{\mathrm{CNO}}=0.0066$. These two models clearly fit the picture given above.

We note that this configuration can be related to the the behaviour seen in Fig. 2 where we see that the central temperature of the radiative-core models reach an upper limit. This is a "thermostat" effect that can be explained by the necessity for models fitting the data to reproduce the luminosity. This ensures that the central temperature of radiative-core models will never reach high levels. In turns, this allow for convective-core models with lower central temperature to have higher central energy generation rates, such as seen in Fig. 3.

Further studies including overshoot but also taking into account out-of-equilibrium nuclear reactions [15] also noticed that small convective cores could be sustained for long times by the pp chain. This is not our case here, since only equilibrium rates were considered.

The last point of interest is that approximately $30 \%$ of the models obtained from our MCMC simulations have almost completely exhausted their central hydrogen. The main region of hydrogen burning is a shell around the centre. As such they can technically be classified as subgiants. Their isothermal cores remain extremely small. However they mark a clear-cut possibility for $\alpha$ Cen A to have already exited the main sequence.

\section{Conclusions}

We focused here on the our latest findings concerning the nuclear reaction process at play at the core of $\alpha$ Cen A. Note that this depends on many other physical prescriptions such as the inclusion of diffusion or the precise formulation of the nuclear reaction rates. Changes in the formulation of these processes can lead to models either certainly convective or radiative in their cores. We refer to [16] for further details.
These results point however towards the need to obtain longer and better seismic data for $\alpha$ Cen $\mathrm{A}$, which never ceases to be an intriguing target for stellar physicists. In particular, it will be necessary to obtain long, continuous and, if possible, precise data. This can be achieved either by the means of space missions (TESS potentially and PLATO) or ground-based networks of telescopes (SONG).

\section{Acknowledgements}

This material is based upon work supported by the NYU Abu Dhabi Institute under grant G1502. Funding for the Stellar Astrophysics Centre is provided by The Danish National Research Foundation (Grant DNRF106).

\section{References}

[1] Eggenberger P. et al., A\&A, 417, 235 (2004)

[2] Kervella P. et al., A\&A, 404, 1087 (2003)

[3] Thoul A. et al., A\&A, 402, 293 (2003)

[4] Bazot M. et al., A\&A, 470, 295 (2007)

[5] Pourbaix D., et al., A\&A, 386, 280 (2002)

[6] Christensen-Dalsgaard J., Ap\&SS, 316, 13 (2008)

[7] Ferguson J. W., ApJ, 623, 585 (2005)

[8] Rogers F. J., Nayfonov A., ApJ, 576, 1064 (2002)

[9] Grevesse, N., \& Noels, Physica Scripta Volume T A. 47, 133 (1993)

[10] Angulo C., et al., Nuclear Physics A 656, 3 (1999)

[11] Böhm-Vitense E., Zeitschrift für Astrophysik 46, 108 (1958)

[12] Haario H. et al., Bernoulli 7, 223 (2001)

[13] Clayton D. D., Principles of stellar evolution and nucleosynthesis (The University of Chicago Press, Chicago, 1984)

[14] Kippenhahn, R., \& Weigert, A., Stellar Structure and Evolution (Springer-Verlag, Berlin Heidelberg New York, 1994) 468

[15] Deheuvels S., et al., A\&A, 514, A31 (2010)

[16] Bazot, M. et al., MNRAS, 460, 1254 (2016) 